\title{
Simulation of the superconducting multiturn flux transformer integrated with a coplanar resonator
}

\author{
H. R. Yi, ${ }^{\text {a) }}$ Y. Zhang, and N. Klein \\ Institut für Schicht- und Ionentechnik (ISI), Forschungszentrum Jülich (FZJ) GmbH, D-52425 Jülich, \\ Germany
}

(Received 21 January 2000; accepted for publication 13 April 2000)

\begin{abstract}
The analysis of the structure of a superconducting multiturn flux transformer integrated with a coplanar resonator for radio-frequency superconducting quantum interference devices is described. Electromagnetic simulations indicate that the loss is dominated by the high loss tangent of the dielectric film used for the separation of the upper and lower superconducting films. The simulated current distribution at its resonant frequency shows that the highest current density is distributed on the multiturn input coil. This current distribution leads to a very high loss when the loss tangent of the dielectric film is high. However, for the same loss tangent of the dielectric film, it is possible to get a reasonably high unloaded quality factor by providing a normal shunt for the multiturn input coil. (ㄷ) 2000 American Institute of Physics. [S0003-6951(00)01624-7]
\end{abstract}

The use of a superconducting coplanar resonator as the tank circuit for radio-frequency (rf) superconducting quantum interference device (SQUID) has improved the magnetic field sensitivity of the single-layer SQUID magnetometer to a level of around $30 \mathrm{fT} / \mathrm{Hz}^{1 / 2}$ on a $10 \times 10 \mathrm{~mm}^{2}$ substrate. ${ }^{1,2}$ The unloaded quality factor $Q_{0}$ of the coplanar resonator patterned from the $\mathrm{YBa}_{2} \mathrm{Cu}_{3} \mathrm{O}_{7}(\mathrm{YBCO})$ film epitaxially grown on the $\mathrm{LaAlO}_{3}(\mathrm{LAO})$ single-crystal substrate was around $20000 .^{2}$ To further improve the field sensitivity, the use a superconducting flux transformer with a multiturn input coil coupled inductively to the SQUID is desirable. However, when the coplanar resonator was integrated with a multiturn flux transformer as shown in Fig. 1(a), the coplanar resonator showed no resonance. ${ }^{3}$ An improved design was proposed in Ref. 4 by Zhang et al. Figure 1(b) shows the schematic layout of this design. The transformer consists of a pickup loop and two input coils, one single turn and another multiturn. The multiturn input coil is shunted by an Au strip. This normal conducting shunt has no effect on the dc supercurrent. A planar double-hole washer SQUID is placed on top of the structure in the flip-chip configuration such that the SQUID holes are coupled to the two input coils. The best measured $Q_{0}$ for this layout was only 600, which was much lower than 20000 for the single-layer coplanar resonator. A high $Q_{0}$ is required so that the condition of $k^{2} Q_{L} \geqslant 1$ for the optimum operation of the rf SQUID can be satisfied. Here, $Q_{L}$ is the loaded quality factor and $k$ is the coupling coefficient between the SQUID and the resonator. In this letter, we performed computer simulations to analyze this integrated trilayer structure, and identified the losses for this system.

We used the electromagnetic analysis software EM developed by Sonnet Software, Inc. ${ }^{5}$ for the simulation. EM calculates $S$ parameters for arbitrary three-dimensional planar passive circuit. A two-port system similar to the experimental one was constructed for the simulations. Two copper loops with outer dimension of $3 \times 3 \mathrm{~mm}^{2}$ and a linewidth of

\footnotetext{
a) Author to whom correspondence should be addressed; electronic mail: h.yi@fz-juelich.de
}

$0.5 \mathrm{~mm}$ were used as the input and output coupling loops. The input loop was placed $5 \mathrm{~mm}$ above the coplanar resonator, and the output loop was placed $5 \mathrm{~mm}$ below the LAO substrate. For each loop, one end was connected to the center conductor of a $50 \Omega$ coaxial transmission cable (i.e., port) and another end was connected to the ground. The coplanar resonator, the pickup loop, and two input coils of the flux transformer were placed on the upper superconducting layer. The connection strip for the multiturn input coil was on the lower superconducting layer. The two superconducting layers were separated by a $300 \mathrm{~nm}$ thick LAO film. The size of the substrate was $10 \times 10 \mathrm{~mm}^{2}$ large and $1 \mathrm{~mm}$ thick. For the LAO single-crystal substrate, the loss tangent of $4 \times 10^{-5}$ and dielectric constant of 24 (Ref. 6) were used for the simulation. The surface resistance of the YBCO film at each frequency was scaled with a quadratic frequency dependence from the reported value at $10 \mathrm{GHz}$ and $77 \mathrm{~K}$ from the literatures ${ }^{7-9}$ i.e., $0.5 \mathrm{~m} \Omega$. The $S$ parameters were simulated at different frequencies. The quality factors were determined from $Q_{1}=f_{0} / \Delta f$, where $f_{0}$ is the resonant frequency and $\Delta f$ is the $3 \mathrm{~dB}$ width of the resonant peak from the transmitted signal $S_{21}$. The unloaded quality factor was calculated from $Q_{0}=2 Q_{1} / \sqrt{10^{S_{11} / 10}}+\sqrt{10^{S_{22} / 10}},{ }^{10}$ where $S_{11}$ and $S_{22}$ were the amplitude in $\mathrm{dB}$ of the reflected signals from ports

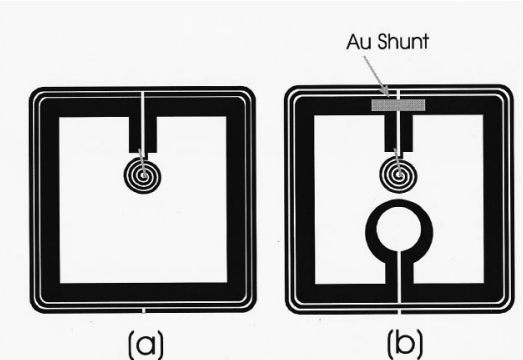

FIG. 1. (a) Normal layout consisting of a coplanar resonator integrated with a multiturn flux transformer; (b) improved layout consisting of a coplanar resonator integrated with a flux transformer having two input coils, one single turn and another multiturn, where a normal conducting shunt is provided for the multiturn input coil. 

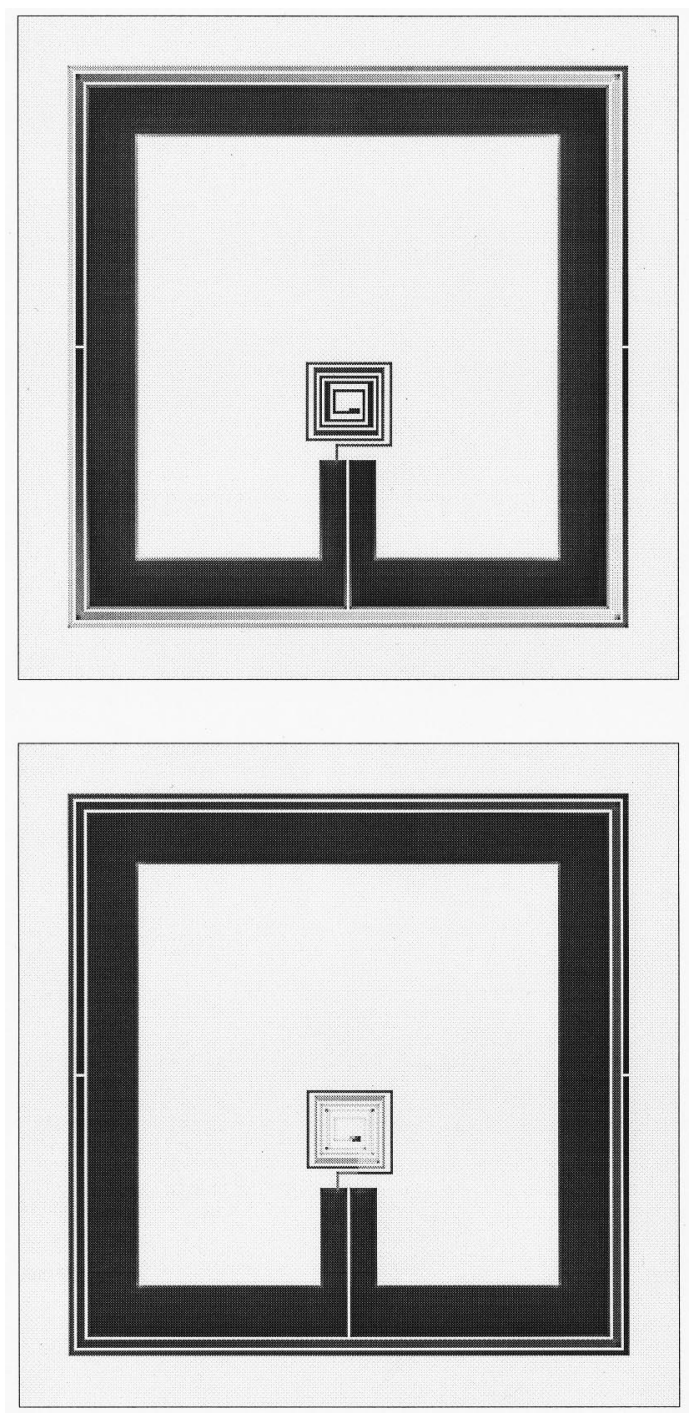

FIG. 2. Simulated current distributions of the normal layout with (lower) and without (upper) the connecting strip for the multiturn input coil. The resonant frequencies are 734 and $657 \mathrm{MHz}$ for the upper and lower parts, respectively. The current scale is from 0 to $1100 \mathrm{~A} / \mathrm{m}$ for the upper part and from 0 to $50 \mathrm{~A} / \mathrm{m}$ for the lower part. The light color represents high current density and the dark color represents low current density.

1 and 2, respectively. The current distribution was simulated at the resonant frequency.

Some approximations have been made in the simulation layout compared to the real layout. As the time required for the calculation is decided by the number of subsections, the available memory of our work station only allows us to use a $50 \times 50 \mu \mathrm{m}^{2}$ unit cell for circuits of $10 \times 10 \mathrm{~mm}^{2}$ size. Therefore, all linewidths less than $50 \mu \mathrm{m}$, e.g., the multiturn input coil, will be replaced by a $50 \mu \mathrm{m}$ linewidth in the simulation. Furthermore, the rounded structures are replaced by the rectangular structures in the simulation.

As reported in Ref. 3, for the normal layout in Fig. 1(b) the resonance was still observed before fabricating the connecting strip. Only after the connecting strip was fabricated forming a closed circuit did the resonance disappear. Figure 2 shows the simulated current distribution at their resonant frequencies for this layout with (lower part) and without (upper part) the connecting strip. The light color represents high current density and the dark color represents low current density. For the simulation layout, the multiturn input coil Downloaded 15 Dec 2006 to 134.94.122.39. Redistribution subject

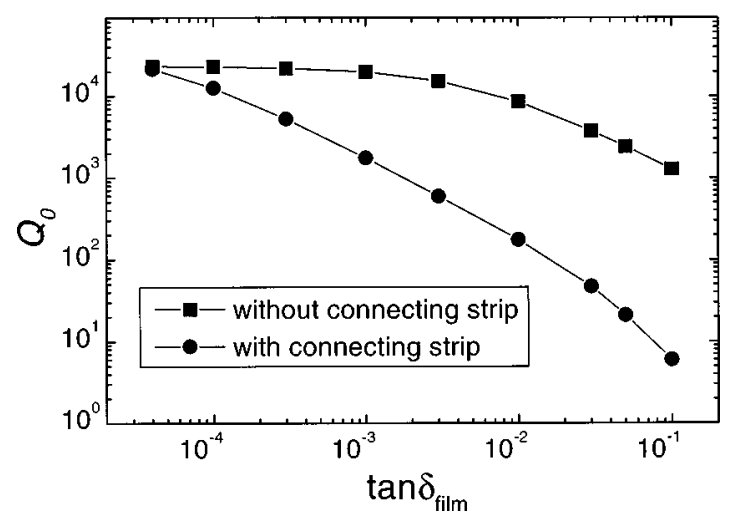

FIG. 3. Simulated unloaded quality factors as a function of the loss tangent of the LAO film for the normal layout with (circles) and without (squares) the connecting strip for the multiturn input coil.

consists of five turns with both the linewidth and the distance between the lines of $50 \mu \mathrm{m}$. Note that for the real layout, the multiturn input coil consists of nine turns with both the linewidth and distance between lines of $20 \mu \mathrm{m}$. Without the connecting strip, the resonant frequency is $734 \mathrm{MHz}$, and the highest current density of about $1100 \mathrm{~A} / \mathrm{m}$ is distributed on the two microstrips of the coplanar resonator, while the highest current density in the multiturn input coil is about 220 $\mathrm{A} / \mathrm{m}$. With the connecting strip, the resonant frequency is $657 \mathrm{MHz}$, and the highest current density of about $50 \mathrm{~A} / \mathrm{m}$ is distributed on the multiturn input coil. Note that the connecting strip in the lower picture is not shown as it is in another YBCO layer. The decrease of the resonant frequency with the connecting strip can be explained by the different current distributions in the two parts of Fig. 2. In the lower picture, a relatively larger portion of resonant current is passing through the multiturn input coil, resulting in a longer effective path for the standing wave of the resonator structure.

The unloaded quality factor describes the total loss in the system. It is composed of the dielectric loss $1 / Q_{d}$, the conduction loss $1 / Q_{c}$, and the radiation loss $1 / Q_{r}: 1 / Q_{0}$ $=1 / Q_{d}+1 / Q_{c}+1 / Q_{r}$. As shown previously, ${ }^{11}$ the radiation loss for such a system is very small $\left(1 / Q_{r}<1.4 \times 10^{-6}\right)$. The conduction loss of this system is also small when YBCO film is used $\left(1 / Q_{c}<1 \times 10^{-5}\right)$. The dielectric loss consists of two parts: one from the LAO substrate and another from the LAO film. The LAO single crystal substrate also has a very low loss tangent. ${ }^{6}$ However, the loss tangent of the dielectric film varies very much depending on the film qualities. ${ }^{12}$ The epitaxial LAO film supports large leakage current. As reported by Pond $e t$ al. ${ }^{13}$ the loss in a trilayer transmission line made from $\mathrm{YBCO} / \mathrm{LAO} / \mathrm{YBCO}$ trilayer structure was primarily limited by conduction currents in the LAO film and damping of the bound charges.

Figure 3 shows the simulated unloaded quality factors for different loss tangent values of the 300-nm-thick LAO film $\left(\tan \delta_{\text {film }}\right.$ ). Note that the loss tangent for the substrate remained unchanged for the simulation, i.e., $4 \times 10^{-5}$. Squares are for the case without the connecting strip and circles are for the case with the connecting strip. In both cases, the simulated $Q_{0} \mathrm{~s}$ are decreasing when $\tan \delta_{\text {film }}$ increases. But $Q_{0}$ decreases much faster with the connecting strip than without it. However, with the connecting strip there is still a resonance $\left(Q_{0}=6\right)$ even for $\tan \delta_{\text {film }}=0.1$, o AIP license or copyright, see http://apl.aip.org/apl/copyright.jsp 


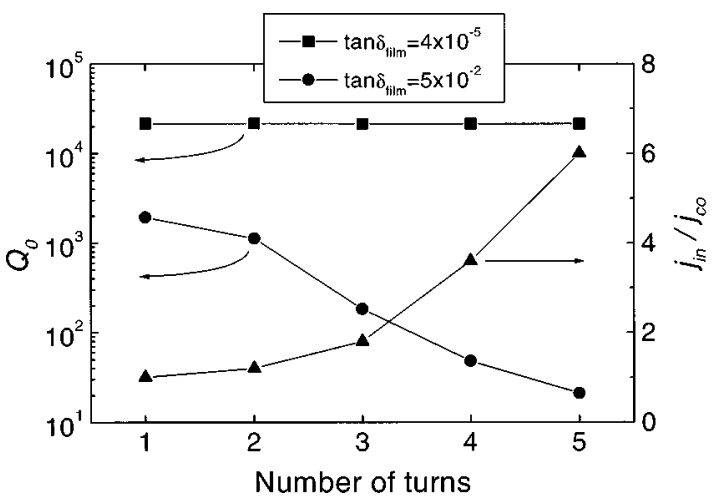

FIG. 4. Simulated unloaded quality factors as a function of the number of turns of the multiturn input coil for the normal layout with the connecting strip. Squares are for $\tan \delta_{\text {film }}=4 \times 10^{-5}$ and circles are for $\tan \delta_{\text {film }}=5$ $\times 10^{-2}$. Triangles show the ratio of the highest current density distributed between the input coil and the coplanar microstrip lines.

while in the experiment, no resonance at all was observed. This is probably due to the approximation made in the simulation, i.e., larger linewidth and fewer turns for the multiturn input coil.

Figure 4 shows the dependence of simulated $Q_{0} \mathrm{~s}$ on the number of turns $n$ of the multiturn input coil for the normal layout. For $\tan \delta_{\text {film }}=4 \times 10^{-5}$ (squares) no decrease of $Q_{0}$ is seen as $n$ increases. However, for $\tan \delta_{\text {film }}=5 \times 10^{-2}$ (circles), $Q_{0}$ drops almost 2 orders of magnitude as $n$ increases from 1 to 5 . Actually, the simulated current distributions for different $n$ showed that the current density on the multiturn input coil becomes higher when $n$ increases. We denote the highest current density on the multiturn input coil as $j_{\text {in }}$, and the highest current density on the coplanar microstrip line as $j_{\text {co }}$. The ratios of $j_{\text {in }} / j_{\text {co }}$ for different $n$ from 1 to 5 are shown by the triangles in Fig. 4 .

Figure 5 shows the simulated current distributions of the improved layout at its resonant frequency of $734 \mathrm{MHz}$. With the normal conducting shunt for the multiturn input coil, the highest current density of about $500 \mathrm{~A} / \mathrm{m}$ is distributed in the two microstrip lines of the coplanar resonator, while the highest current density in the multiturn input coil is only 8 $\mathrm{A} / \mathrm{m}$. The latter number is considerably lower than $50 \mathrm{~A} / \mathrm{m}$ for the normal layout in the lower part of Fig. 2, which indicates that the normal conducting shunt for the multiturn input coil is indeed effective. For this layout, the simulated unloaded quality factors also decrease when $\tan \delta_{\text {film }}$ increases. However, unlike the normal layout, one still gets reasonably high $Q_{0}$ s (i.e., $1000-3000$ ) for $\tan \delta_{\text {film }}$ $=0.03-0.1$. The simulated $Q_{0}$ s are somewhat higher than that measured from the experiment, possibly because of the approximation made in the simulation.

In summary, we have analyzed the trilayer structure of

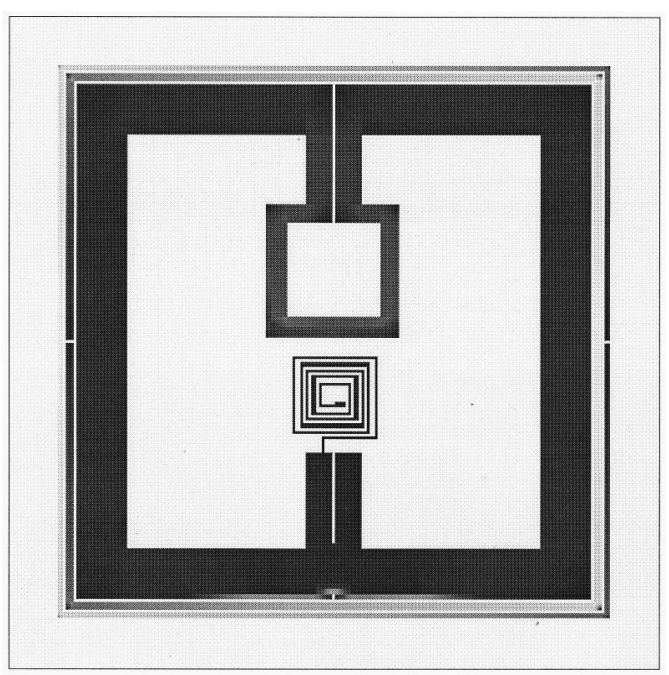

FIG. 5. Simulated current distribution of the improved layout at its resonant frequency of $734 \mathrm{MHz}$. The current scale is from 0 to $500 \mathrm{~A} / \mathrm{m}$.

the superconducting coplanar resonator integrated with a multiturn flux transformer for the rf SQUID operation. Computer simulations show that the loss is dominated by the high loss tangent of the dielectric film. For the normal layout, the highest current density is distributed on the multiturn input coil. This current distribution leads to a very high loss when the loss tangent of the dielectric film is high. However, for the same loss tangent of the dielectric film, it is possible to get a reasonably high unloaded quality factor by providing a normal shunt for the multiturn input coil. These simulated results agree well with the experimental results.

The authors gratefully acknowledge A. I. Braginski for stimulating discussions.

${ }^{1}$ Y. Zhang, W. Zander, J. Schubert, F. Rüders, H. Soltner, M. Banzet, N. Wolters, X. H. Zeng, and A. I. Braginski, Appl. Phys. Lett. 71, 704 (1997).

${ }^{2}$ Y. Zhang, N. Wolters, X. H. Zeng, J. Schubert, W. Zander, H. Soltner, H. R. Yi, M. Banzet, F. Rüders, and A. I. Braginski, Appl. Supercond. 6, 385 (1998).

${ }^{3}$ H. R. Yi, J. Schubert, W. Zander, X. H. Zeng, M. Banzet, D. F. He, and Y. Zhang, Semicond. Sci. Technol. 11, 692 (1998).

${ }^{4}$ Y. Zhang, H. R. Yi, J. Schubert, W. Zander, M. Banzet, and A. I. Braginski, Appl. Phys. Lett. 72, 2029 (1998).

${ }^{5}$ Sonnet Software, Inc. Liverpool, NY.

${ }^{6}$ G. A. Samara, J. Appl. Phys. 68, 4214 (1990).

${ }^{7}$ N. Klein, G. Müller, H. Piel, B. Roas, L. Schultz, U. Klein, and M. Peiniger, Appl. Phys. Lett. 54, 757 (1989).

${ }^{8}$ K. H. Young and J. Z. Sun, J. Appl. Phys. 70, 3748 (1991).

${ }^{9}$ N. Newman, B. F. Cole, S. M. Garrison, K. Char, and R. C. Taber, IEEE Trans. Magn. 27, 1276 (1991).

${ }^{10}$ N. Tellmann, Ph.D. thesis, University of Wuppertal, Wuppertal, 1996.

${ }^{11}$ H. R. Yi, Y. Zhang, and A. I. Braginski, Appl. Phys. Lett. 73, 2357 (1998).

${ }^{12} \mathrm{X}$. X. Xi (private communications).

${ }^{13}$ J. M. Pond, K. R. Carroll, J. S. Horwitz, D. B. Chrisey, M. S. Osofsky, and V. C. Cestone, Appl. Phys. Lett. 59, 3033 (1991). 